\title{
PREDICTIVE AND PROGNOSTIC MODELLING AND SIMULATION OF COATINGS SUBJECT TO CORROSION AND MECHANICAL FAILURES
}

\author{
ZULFIQAR AHMAD KHAN ${ }^{1}$, JAWWAD LATIF ${ }^{1}$, HAMMAD NAZIR $^{1}$, ADIL SAEED ${ }^{2} \&$ KEITH STOKES ${ }^{3}$ \\ ${ }^{1}$ NanoCorr, Energy \& Modelling (NCEM), Department of Design and Engineering, Faculty of Science \& \\ Technology, Bournemouth University, United Kingdom. \\ ${ }^{2}$ Global College of Engineering and Technology, Muscat Sultanate Oman. \\ ${ }^{3}$ University of Southampton, National Centre for Advanced Tribology at Southampton (nCATS) \\ Faculty of Engineering and the Environment, Southampton.
}

\begin{abstract}
This research presents analytical and mathematical modelling of coating failures within industrial components, structures, mobile assets and systems due to corrosive degradation and mechanical fracture. These failures lead to several surface problems; therefore, contact mechanics and electrochemistry approaches incorporating induced residual stresses have been adopted to develop a comprehensive solution for the prediction and prognostic of such failures. Experimental study of film cracking and its propagation into substrates, interfacial transient behaviours and film-substrate system has been conducted. A parallel study of corrosive degradation to include cathodic delamination, cathodic blistering and tribo-corrosion of films has been conducted. Experimental and analytical studies of induced residual stresses within the coating and their effects on failure mechanisms and propagation have been completed. A detailed investigation of elastic mismatch at the interfacial contact and interfacial crack tip field has been performed and a complex stress intensity factor is presented. Mathematical derivation of oscillatory singularity, mode mix and interfacial fracture criterion to include adhesion are presented. This paper presents novel mathematical modelling incorporating interfacial crack propagating, diffusion of corrosive species and cathodic blistering for prediction and prognoses of coating failures.

Keywords: coating failure, compressive residual stress, diffusion induced stress.
\end{abstract}

\section{INTRODUCTION}

Protective coatings on industrial components and structures act as a barrier to preserve them from chemical and physical attack. Various environmental and mechanical factors are involved in the breakdown of coating on metallic surfaces. Defects in coating and substrate are formed during the fabrication process and during the application of system. The difference in coefficient of thermal expansion between coating and the substrate is the major cause of producing micro-cracks in coating. Cracks can also occur due to abrasion, physical or mechanical contact. The presence of residual stresses in coating and substrate system causes compressive and tensile stress. In corrosive environment, tensile residual stress results in crack opening, which leads to stress corrosion cracking. Micro-cracks provide passageway to corrosive species to diffuse into substrate and cause electrochemical reactions which results in coating delamination [1]. However, compressive residual stress closes the micro-cracks, but it causes the blister delamination when these stresses are sufficiently high [2]. Compressive residual stress and diffusion induced stress play a major role in adhesion loss of coating from the substrate. Various mathematical models have been formulated to model these two parameters for reliability assessment. Current work presents predictive modelling for the propagation of coating deformation in the presence of micro-cracks as a function of compressive residual and diffusion induced stresses. 


\section{BACKGROUND}

A model for de-bonding driving force as a function of stress to predict the duration of useful life of isotropic coatings is presented in [2]. Elastic modulus and residual stress play a major role in the formation and propagation of blisters [3]. Stress-driven viscoplastic deformation conditions were also proposed to explain blistering mechanism [4]. Modified model to explain the deformation induced by residual stress for multilayer structure has been proposed in [5]. Later on, closed form solutions have been proposed in [6-8] by incorporating residual stress parameter. Diffusion induced stress factor is also a major cause for coating delamination, which cannot be neglected and has drawn considerable interest of researchers in the past decade. Various mathematical models have been proposed to model the concentration and diffusion of corrosive species inducing stress in the system. The effects of diffusion stress has been investigated in [9]. A theory has been presented in [10] that describes the delamination initiation when ions migrate through conductive passages which are usually formed in the presence of water molecules in hydrophilic and low molecular weight regions. Prognostic model designed in [10] was a function of blister size, ion diffusivity, potential gradient and distance between defect and blister. Diffusion induced stress is also a major problem in lithium ion batteries. The surface energy has been modelled by merging diffusion induced stress problem [11]. Considering diffusion induced stress and residual stress as important delamination parameters, it is very important to investigate and model the coupling behaviour of both factors.

Nanocorr, Energy and Modelling (NCEM) research group has been investigating corrosion and coating failure problems in isotropic organic coatings. Various parameters have been investigated and modelled to explain the behaviour of corrosion and coating failures [12-25]. Recently, the coupling effects of compressive residual stress and diffusion induced stress have been investigated through experimental analysis to model the formation and propagation of circular blisters in the presence of micro-cracks over coated surface. In this research, the de-bonding driving forces for straight-sided blister are modelled to explain blister formation and propagation mechanism in the presence of micro-crack.

\section{EXPERIMENTAL ANALYSIS}

In order to analyse the effect of compressive residual stress and diffusion induced stress, corrosion test was applied on coated samples having micro-crack over coating [16]. Seven samples were used to obtain cross-sectional measurements. AISI-1020 carbon steel samples $0.18 \%-0.23 \% \mathrm{C}, 0.3 \%-0.6 \% \mathrm{Mn}$ with balanced Fe were used for experimental analysis as substrates. The thicknesses of samples were $0.01 \mathrm{~cm}$ with elastic modulus of $200 \mathrm{GPa}$ and coefficient of thermal expansion as $11.7 \times 10^{-6} \mathrm{~K}^{-1}$ [26]. Sample were polished with emery paper of grit size 200 then conditioned with distilled water and dried with cold air.

Spray gun was used to apply red oxide primer coating on samples. The elastic modulus of red-oxide is $6.14 \mathrm{GPa}$ with coefficient of thermal expansion as $21.6 \times 10^{-6} \mathrm{~K}^{-1}$. Coating thickness was $20 \mu \mathrm{m}$. Micro-cracks were induced over the coating surface with initial aperture size of $2.1 \mu \mathrm{m}$. Residual stresses were measured through charge coupled device (CCD) detector and laser optics, which measures stress by monitoring substrate curvature. When samples were exposed to corrosive environment and monitored through the microscope, it was observed that several blisters and micro-cracks started to appear over the surface of coated samples. All the micro-cracks have shown almost similar behaviour but single microcrack from each sample is considered for cross-sectional observation and analysis which is a destructive method. 
The purpose of experimental work was to analyse the effects of residual induced stress in presence of diffusion induced stress. Samples were kept at fabrication temperature of $318 \mathrm{~K}$ then transported to corrosive atmospheric chamber for accelerated testing. The fog, 5 parts of $\mathrm{NaCl}$ in 95 parts of distilled water, was simulated. The samples were placed at $60^{\circ} \mathrm{C}$ to avoid any direct pathways of crystallisation into the coating. The corrosive atmosphere was accelerated by gradually increasing the temperature. The temperature was raised $1 \mathrm{~K}$ for every one hour. Cross-sectional images of each sample were taken during the temperature values i.e. 319K, 321K, 328K, 333K, 353K. During microscopic analysis, temperature is maintained similar to that in the corrosion simulation chamber for precision. Diffusion induced stress was induced by the presence of corrosive species in the environmental chamber and residual stress was induced by the change in temperature due to elastic mismatch. Micro-cracks provide channels for the species to diffuse into the system. Tensile and compressive behaviour of residual stress controls the opening and closing of pre-existing micro-cracks in the coating. The experimental compressive residual stress and diffusion induced stress with respect to change in temperature are shown in Fig. 2. The investigation of micro-crack propagation is divided into three regions and the resultant stress for controlling blister propagation was derived.

\section{Region 1:}

The experiment was initiated from fabrication temperature $(318 \mathrm{~K})$ of system and increased to $319 \mathrm{~K}$. The aperture size of micro-crack was measured to be $2.1 \mu \mathrm{m}$, as shown in Fig. 1(a), which provided channel for the corrosive species to diffuse into the system, as a result the

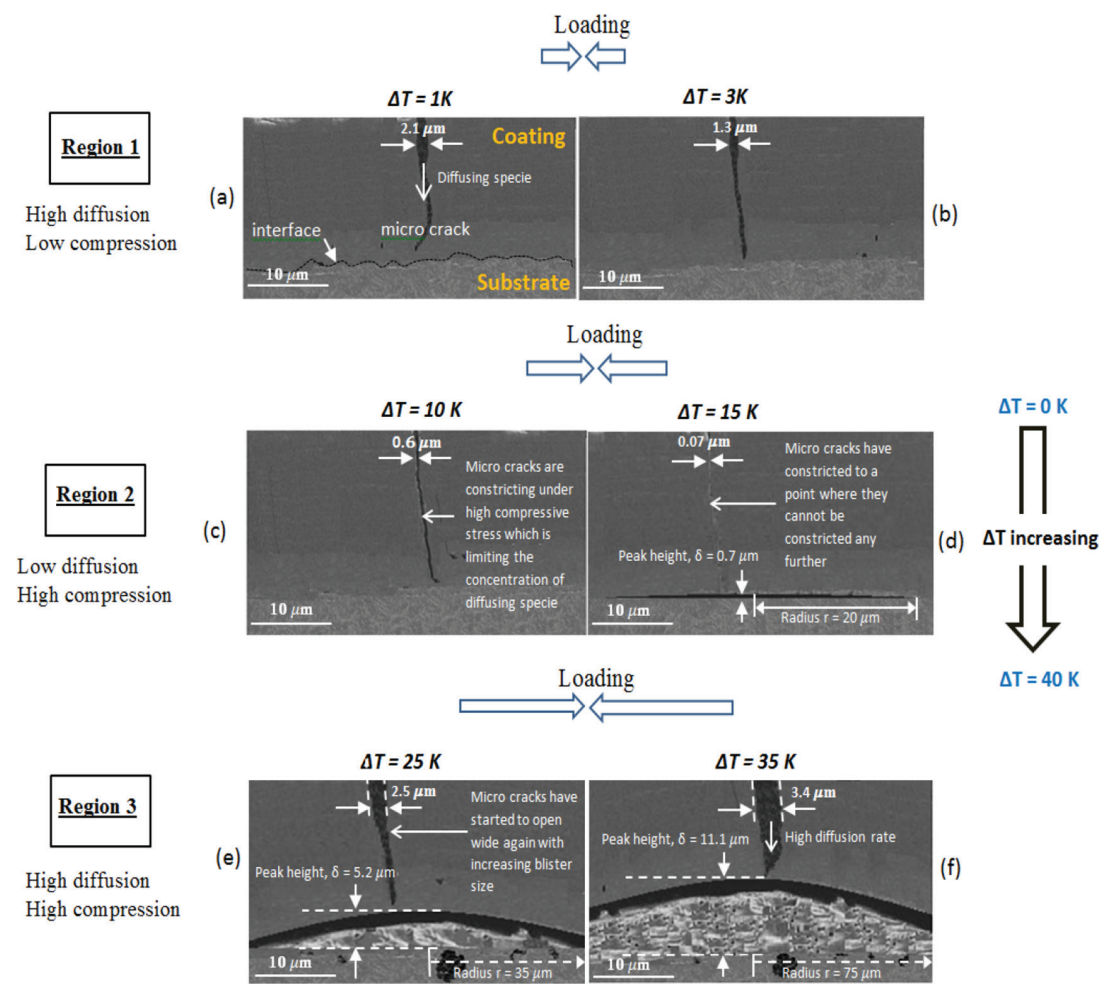

Figure 1: Samples micrographs at various temperature values [16]. 


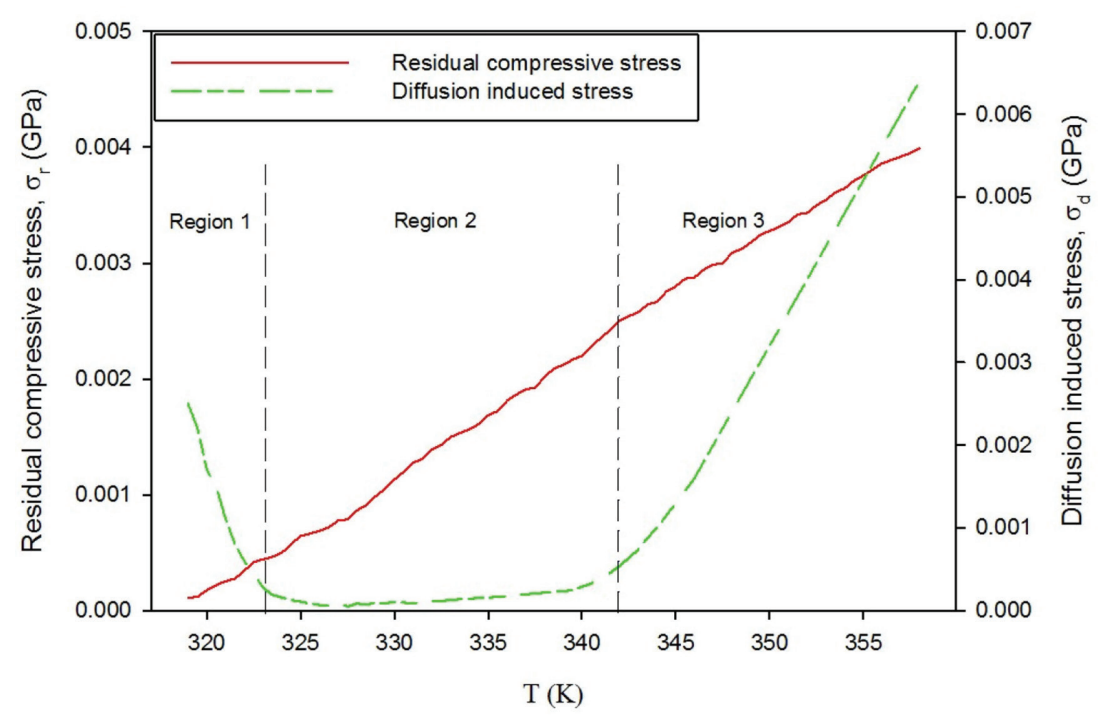

Figure 2: Experimental results of residual compressive stress and diffusion induced stress [16].

diffusion stress was high at the beginning of the experiment, as shown in Fig. 2. When the temperature is further increased to $321 \mathrm{~K}$ the micro-crack started to shrink due to the rise in compressive residual stress but no major blistering effect was observed. The aperture of micro-crack decreased to $1.3 \mu \mathrm{m}$, as shown in Fig. 1(b). Small decrease in diffusion induced stress was observed due to minor rise in temperature. Diffusion induced stress in this region appears to be a major controlling factor. According to the theory of linear superposition the following relation can be concluded.

$$
\sigma=\sigma_{r}+\sigma_{d} \cong \sigma_{d}
$$

\section{Region 2:}

When the temperature was raised to $328 \mathrm{~K}$, the aperture size of micro-crack decreased to $0.6 \mu \mathrm{m}$. The reason of this decrease in the size is the increase in compressive residual stress that can be seen in the results, which are plotted in Fig. 2. The compressive residual stress continued to increase from $0.004 \mathrm{GPa}$ to $0.0023 \mathrm{GPa}$ in region 2 . The decrease in channel size for the corrosive species to diffuse into the system also decreased the diffusion induced stress. Further increase in the temperature to $331 \mathrm{~K}$, increased the compressive residual stress which reduced the aperture size to $0.07 \mu \mathrm{m}$. This increase in compressive residual stress initiated the blistering effect, which can be seen in Fig. 1(d). Residual compressive stress can be considered as a significant influential parameter and can be written as:

$$
\sigma=\sigma_{r}+\sigma_{d} \cong \sigma_{r}
$$

\section{Region 3:}

When the temperature was increased to $341 \mathrm{~K}$, compressive residual stress increased significantly which leads to blistering delamination. The size of the blister increased thus making the aperture size of micro-crack to further increase to $2.5 \mu \mathrm{m}$. The widening of micro-crack provided more space for corrosive species to diffuse into the system, hence diffusion induced stress started to rise after $341 \mathrm{~K}$. In region 3 , the behaviour of increase in compressive residual stress and diffusion induced stress was observed to be identical. The amplitude and radius of 
blister as observed was $5.2 \mu \mathrm{m}$ and $35 \mu \mathrm{m}$, which continually increased and reached up to a radius as $75 \mu \mathrm{m}$ and amplitude as $11 \mu \mathrm{m}$. After applying the principle of linear superposition on coupling effect of compressive residual stress and diffusion induced stress for blister growth and propagation, the following relation can be derived as:

$$
\sigma=\sigma_{r}-\sigma_{d} .
$$

The relation of compressive residual stress and diffusion induced stress proposed in eqn (3) is considered as a major controlling factor in blistering delamination mechanism. The coupling effect relation for compressive residual and diffusion induced stress has been previously incorporated for de-bonding driving force of circular blister growth and propagation. In current research the de-bonding driving force for straight-sided blister growth and propagation is proposed. Modelling of circular blister and straight-sided blister is derived in the next section.

\section{MATHEMATICAL MODEL}

The coupling effect of compressive residual stress and diffusion induced stress has been investigated through experimentation and the relation for stress as a function of compressive residual stress and diffusion induced stress has been proposed by using the principle of linear superposition theory. This paper discusses the incorporation of observed stress behaviour in circular blister propagation and based on that theory, relations for de-bonding force of straight-sided blisters are also proposed. Multi-disciplinary modelling approach has been adopted to explain the effect of stresses in coating delamination process. The designed and proposed models are based on the concepts of thermodynamics, mechanics and bi-layer cantilever beam theory. The critical stress level in the system can be calculated by using the following relation [2]:

$$
\sigma_{c r}=1.2235 \frac{E}{\left(1-v^{2}\right)}\left(\frac{h}{r}\right)^{2}
$$

Here, $\sigma_{c r}$ is the critical stress, which computes critical threshold for the initiation of delamination, $\mathrm{h}$ is coating thickness, $\mathrm{r}$ is radius of blister, $\mathrm{E}$ is elastic modulus and $v$ poisson's ratio. The coupling effect of compressive residual stress and diffusion induced stress controlling the transport of corrosive species as observed through experimental analysis [16] can be written as follows:

$$
\sigma_{0}=\frac{\partial \sigma_{r_{c}}}{\partial x}-\frac{\partial \sigma_{d_{c}}}{\partial t},
$$

$\sigma_{0}$ represents total stress according to linear superposition theory, $\frac{\partial \sigma_{r_{c}}}{\partial t}$ is the change in compressive residual stress with respect to location $\mathrm{x}$ and $\frac{\partial \sigma_{d_{c}}}{\partial t}$ is the change in diffusion induced stress with respect to time $t$, change in concentration of corrosive species as a function of environmental parameters and the coupling effects of diffusion induced and compressive stress was derived [16] as:

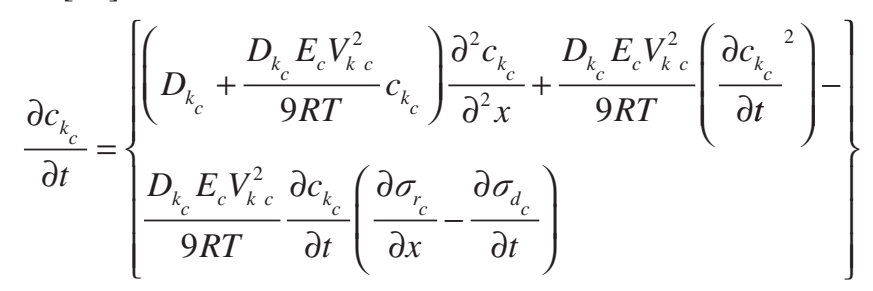


$\frac{\partial c_{k_{c}}}{\partial t}$ represents a change in the concentration of corrosive species on coating and substrate system through micro-cracks, $D_{k_{c}}$ diffusion coefficient of coating, $\mathrm{T}$ is temperature, $\mathrm{R}$ is molar gas constant and $V_{k_{c}}$ is partial molar volume of coating material. Coupling effect of stress using eqn (2) can be written as:

$$
\sigma_{0}=\frac{\partial \sigma_{r_{c}}}{\partial x}-\frac{\partial \sigma_{d_{c}}}{\partial t}=\left(D_{k_{c_{i}}}+\frac{D_{k_{c}} E_{c} V_{p k_{c}}^{2}}{9 R T}\right) \frac{\partial^{2} c_{k_{c}}}{\partial^{2} x}+\frac{D_{k_{c}} E_{c} V_{p k_{c}}^{2}}{9 R T}\left(\frac{\partial c_{k_{c}}^{2}}{\partial t}\right)\left(\frac{D_{k_{c}} V_{p k_{c}}^{2}}{9 R T} \frac{\partial c_{k_{c}}}{\partial t}\right)^{-1}
$$

\subsection{De-bonding driving force for circular blister}

When coating remains intact with the substrate, there is no de-bonding driving force. The blistering effect appears when de-bonding driving force exceeds critical threshold value. The blisters shapes can be circular which end up in telephonic cord like shape and blister can also lead to straight-sided shape which ends up as snakes back forth shape [27]. In this research paper the coupling effect of compressive residual stress and diffusion induced stress, which was investigated for circular blister through experimental results, is incorporated for straightsided blister to model de-bonding driving force [16]. First, the derivation of de-bonding driving force for circular blister is discussed and then, relations for straight-sided blisters are proposed. The model for circular blister is already analysed and validated through experiments while strain energy release rate for straight-sided blister has already been discussed and validated $[16,27]$. So, new relations for straight side blister are based on two verified and validated theories. Strain energy release rate stored for circular blister can be written as:

$$
G_{0}=\frac{(1-v) h \sigma^{2}}{E} .
$$

Strain energy release rate for crack propagation derived in Ref. [2] is given as:

$$
G=6 \frac{\left(1-v^{2}\right)}{E h^{3}}\left(M^{2}+\frac{1}{12} h^{2} \Delta N^{2}\right) \text {. }
$$

$\mathrm{G}$ represents strain energy release rate for circular blister propagation, $\mathrm{M}$ is bending moment and $N$ is resultant stress force $\left(\sigma=\sigma_{r}-\sigma_{d}\right)$ acting on coating. By incorporating the coupling effect of compressive residual stress and diffusion induced stress in crack propagation can be derived as:

$$
G^{\prime}=6 \frac{\left(1-v^{2}\right)}{E h^{3}} M^{2}\left(1+\frac{1}{4}\left(\sqrt{\left.\left(\frac{\partial \sigma_{r_{c}}}{\partial x}-\frac{\partial \sigma_{d_{c}}}{\partial t}\right) \sigma_{c r}^{-1}-1\right)}\right)^{2}\right)
$$

The term $\cap=\left(\frac{\partial \sigma_{r_{c}}}{\partial t}-\frac{\partial \sigma_{d_{c}}}{\partial t}\right) \sigma_{c r}{ }^{-1}$ is de-bonding index which controls blister formation and propagation. By using relation of mode dependent strain energy release rate the following model can be formulated for de-bonding driving force. 


$$
F=6 \frac{\left(1-v^{2}\right)}{E h^{3}} M^{2}\left(1+\frac{1}{4}(\sqrt{\bigcap-1)})^{2}\right)\left(\sec ^{2}\left[\left(1-\frac{\Gamma_{\mathrm{IC}}}{E_{c} \lambda}\right) \psi\right]\right)^{-1}
$$

F represents de-bonding driving force, $\Gamma_{\mathrm{IC}}$ is coating toughness, $\lambda$ is roughness of substrate interface and $\psi$ is the ratio of coating crack edge from mode II to mode I.

\subsection{De-bonding driving force for straight-sided blister}

The derivation of de-bonding driving force for straight-sided blister propagation is proposed and derived in this section. The straight-sided blister proliferates after the complete formation of curved 'front' of blister. The straight-sided blister is thought to provide accurate measure of primary area of de-bonded interface needed to initiate buckle delamination along with energy release rate [27]. The strain energy release rate [27] in direction parallel to crack front can be written as:

$$
G_{0}=\frac{\left(1-v^{2}\right)}{2 E} \sigma_{0}^{2} h
$$

By incorporating the $\sigma_{0}=\frac{\partial \sigma_{r_{c}}}{\partial t}-\frac{\partial \sigma_{d_{c}}}{\partial t}$ stress, which has been concluded in previous section in the above equation, the strain energy release rate for straight-sided blister due to coupling effect of compressive residual stress and diffusion induced stress can derived as:

$$
\mathrm{G}_{0}^{\prime}=\frac{\left(1-v^{2}\right) h}{2 E}\left(\frac{\partial \sigma_{r_{c}}}{\partial t}-\frac{\partial \sigma_{d_{c}}}{\partial t}\right)^{2}
$$

In straight-sided blister, the energy release rate relation for crack propagation at 'side' and 'front' has separate relations [27]. The 'side' and 'front' sides of straight-sided blister is labelled in Fig. 3. For blister side strain energy as a function of stresses can be written as:

$$
G_{\text {side }}=G_{0}\left(1-\frac{\sigma_{c}}{\sigma_{0}}\right)\left(1+3 \frac{\sigma_{c}}{\sigma_{0}}\right)
$$

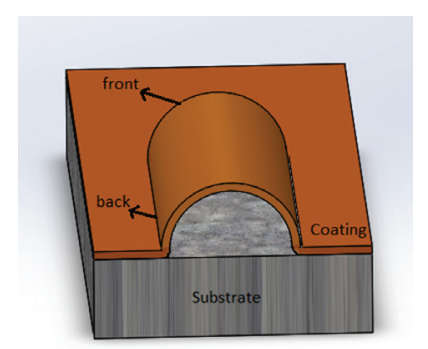

Figure 3: Schematic of straight-sided blister. 
By incorporating the $\sigma_{0}=\frac{\partial \sigma_{r_{c}}}{\partial t}-\frac{\partial \sigma_{d_{c}}}{\partial t}$ in the above relation the strain energy for 'side' of blister as a function of coupling effect of compressive residual stress and diffusion induced stress can be written as:

$$
G_{\text {side }}^{\prime}=\frac{\left(1-v^{2}\right) h}{2 E}\left(\frac{\partial \sigma_{r_{c}}}{\partial t}-\frac{\partial \sigma_{d_{c}}}{\partial t}\right)^{2}\left(1-\sigma_{c}\left(\frac{\partial \sigma_{r_{c}}}{\partial t}-\frac{\partial \sigma_{d_{c}}}{\partial t}\right)^{-1}\right)\left(1+3 \sigma_{c}\left(\frac{\partial \sigma_{r_{c}}}{\partial t}-\frac{\partial \sigma_{d_{c}}}{\partial t}\right)^{-1}\right)
$$

De-bonding driving force for the 'side' of blister as a function of mode mix can be derived as:

$$
F_{\text {side }}=\frac{\left(1-v^{2}\right) h}{2 E} \sigma_{0}^{2}\left(1-\bigcap^{-1}\right)\left(1+3 \bigcap^{-1}\right)\left(\sec ^{2}\left[\left(1-\frac{\Gamma_{\mathrm{IC}}}{E_{c} \lambda}\right) \psi\right]\right)^{-1} \text {. }
$$

$F_{\text {side }}$ is the de-bonding driving force for 'side' of straight-sided blister, which is a function of mod mix and de-bonding index. Similarly, the de-bonding driving force for 'front' of blister can be derived as follows:

$$
G_{\text {front }}=G_{0}\left(1-\frac{\sigma_{c}}{\sigma_{0}}\right)
$$

By including de-bonding index in eqn (13), it can be modified as:

$$
G_{\text {front }}^{\prime}=\frac{\left(1-v^{2}\right) h}{2 E}\left(\frac{\partial \sigma_{r_{c}}}{\partial t}-\frac{\partial \sigma_{d_{c}}}{\partial t}\right)^{2}\left(1-\sigma_{c}\left(\frac{\partial \sigma_{r_{c}}}{\partial t}-\frac{\partial \sigma_{d_{c}}}{\partial t}\right)^{-1}\right)
$$

De-bonding driving force for the 'front' of blister as a function of mode mix can be derived as:

$$
F_{\text {front }}=\frac{\left(1-v^{2}\right) h}{2 E} \sigma_{0}^{2}\left(1-\bigcap^{-1}\right)\left(\sec ^{2}\left[\left(1-\frac{\Gamma_{\mathrm{IC}}}{E_{c} \lambda}\right) \psi\right]\right)^{-1}
$$

The 'front' and 'side' de-bonding driving forces for straight-sided blister are proposed by incorporating the coupling effect of compressive residual stress and diffusion induced stress.

\section{SIMULATION RESULTS}

\subsection{Simulation results for circular blister}

The resultant stress force due to coupling effect of compressive residual stress and diffusion induced stress proposed for circular blister formation and propagation has been validated which shows strong agreement between experimental observation and mathematical modelling [16]. Experimental and simulation results for normalised de-bonding driving force as a function of change in temperature are shown in Fig. 4. All parameters are converted into dimensionless form for simulation of normalised de-bonding driving force. In region 1, the coating and substrate system was in safe condition and there was no change in pre-existing micro-crack and de-bonding driving force was also zero. In region 2, micro-crack 


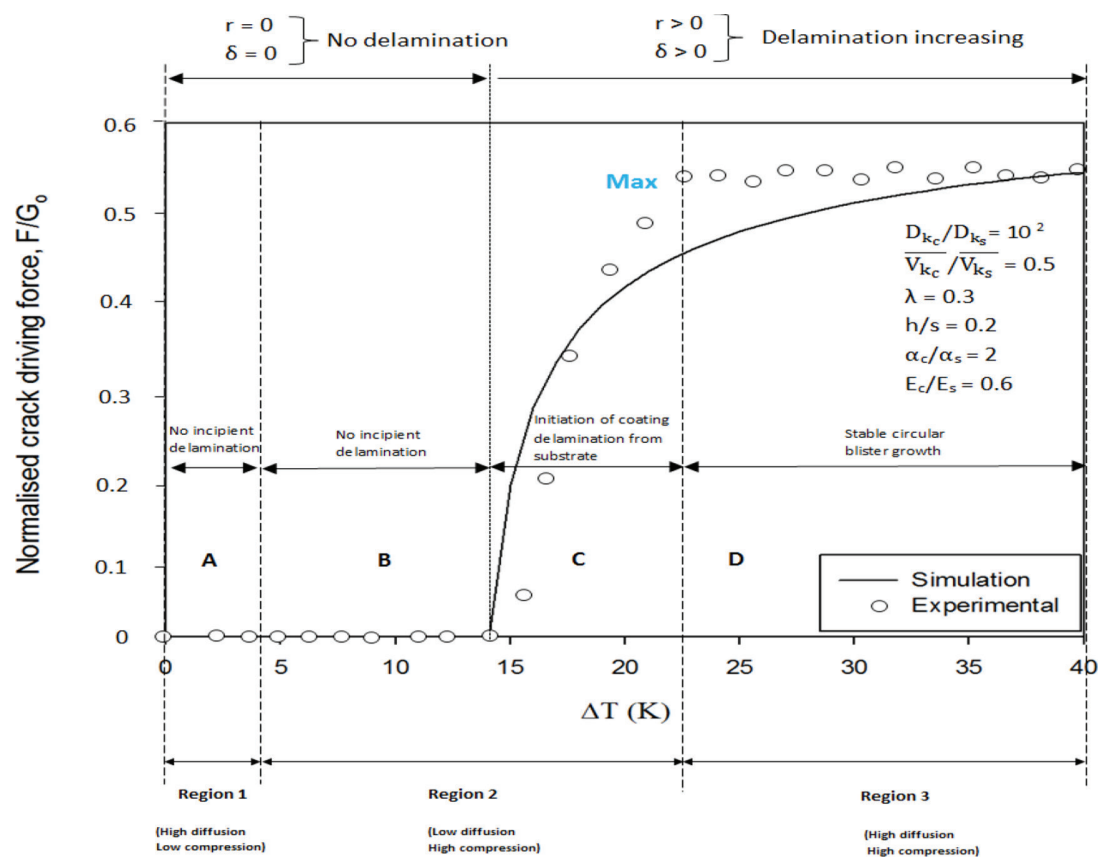

Figure 4: Simulation and experimental results of de-bonding driving force for circular blister [16].

aperture size started to reduce due to high compression rate. Diffusion rate decreased due to the reduction in aperture size of micro-crack. Normalised de-bonding driving force was zero till $\Delta \mathrm{T}=14 \mathrm{~K}$; hence, coating can be considered in safe condition. Further increase in temperature resulted in adhesion loss in the form of blistering and de-bonding driving force continued to increase and reach at maximum level. In region 3, compressive residual stress continues to increase and the behaviour of diffusion rate seems similar to compressive residual stress. In this region the size of blister increased significantly and micro-crack also expanded.

\subsection{Simulation results for straight-sided blister}

The proposed mathematical models for straight-sided blister is based on validated theory as explained in previous sections $[16,27]$. The simulation result of straight-sided blister is shown in Fig. 5. The mathematical equations for simulating de-bonding driving forces for 'side' and 'front' were converted into normalised form. It is worth noting that the de-bonding driving force for the 'side' of straight-sided blister is always greater as compared to the de-bonding driving force for the 'front' but crack propagates at the 'front'. The reason is that the 'front' experiences lower effective interface toughness [27]. For sufficiently wide straight-sided blister, the energy release rate along curved front exceeds than that of 'side' for large (by a factor of ten or more) coating modulus than substrate which would allow propagation of straight-sided blisters despite of interface toughness is mode independent [28]. 


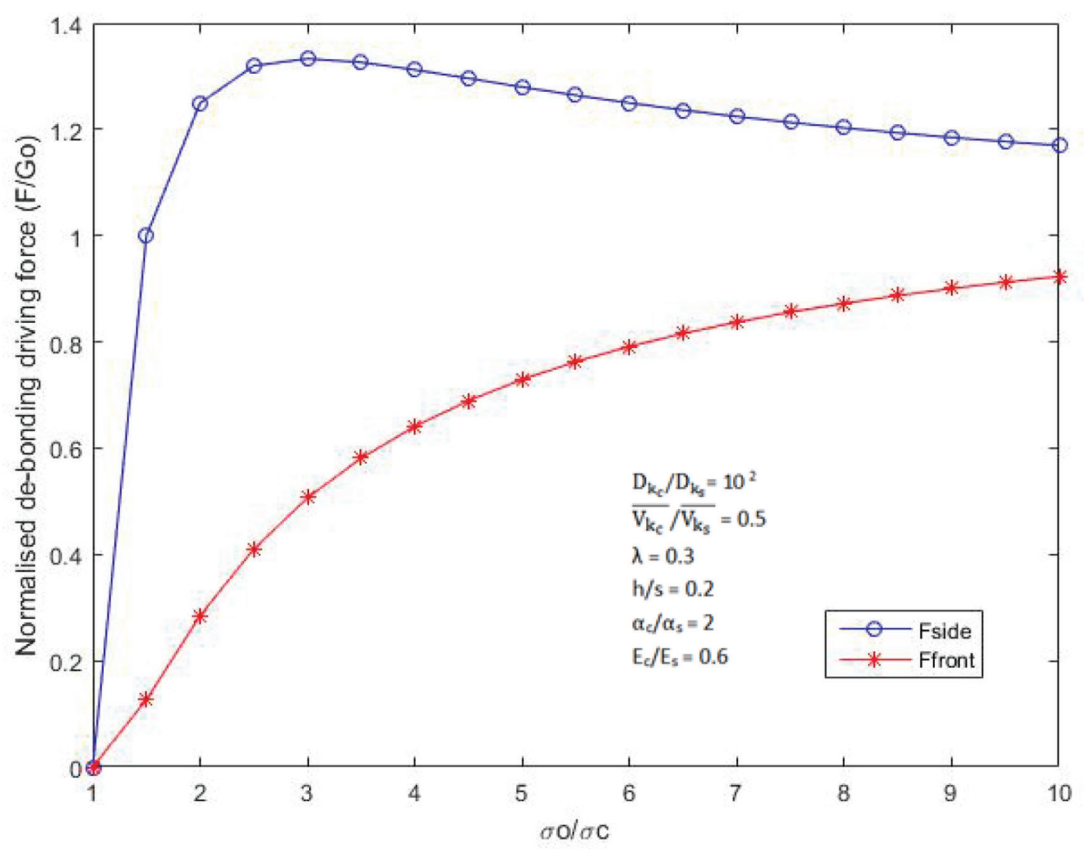

Figure 5: Simulation results of de-bonding driving forces for straight-sided blister.

\section{CONCLUSIONS}

This paper presents the mathematical modelling for circular and straight-sided blister growth and propagation by incorporating coupling effect of compressive residual stress and diffusion induced stress. The effects of stresses are investigated in the presence of micro-cracks. The difference in coefficient of thermal expansion between coating and substrate system induced compressive residual stress which continued to increase due to an increase in temperature while the corrosive species produced diffusion induced stress. Initially, increase in temperature caused pre-existing micro-crack to shrink and reduce diffusion induced stress while further increase in temperature caused de-bonding driving force to exceed its critical level which resulted in blister formation and it started propagating in circular shape. The rising trend of diffusion induced stress appears to be similar as compressive residual stress at high temperature. Based on experimental observations, resultant stress for blister formation and growth is proposed and incorporated in circular and straight-sided blisters formation and propagation to propose the de-bonding driving forces. De-bonding driving forces are proposed for blisters growth and propagation which can be used for coating life assessment. Proposed mathematical models can be further extended for 'telephonic cord' which appears after the propagation of circular blister and for 'back and forth' shape which appears after the propagation of straight-sided blister. Comprehensive analyses of circular blister formation and propagation has been conducted through experimentation and by simulating the effects of various parameters (modulus of elasticity, coefficient of thermal expansion, coating thickness and poisson's ration) in previous NCEM papers. In future, further analysis of proposed de-bonding driving forces of straight-sided blisters will be presented.

\section{ACKNOWLEDGEMENT}

This research is joint funded by the Defence Science and Technology Laboratory (DSTL), Ministry of Defence (MoD) and Bournemouth University UK. The authors acknowledge their financial support and in kind contributions. 


\section{REFERENCES}

[1] Parkins, R., Stress Corrosion Cracking, Uhlig's Corrosion Handbook, p. 191, 2011. https://doi.org/10.1002/9780470872864.ch14

[2] Hutchinson, J., Thouless, M. \& Liniger, E., Growth and configurational stability of circular, buckling-driven film delaminations. Acta metallurgica et materialia, 40(2), pp. 295-308, 1992. https://doi.org/10.1016/0956-7151(92)90304-W

[3] Chuang, T. \& Nguyen, T., A non-osmotic blister growth model in coating systems. in damage and failure of interfaces. 1st International Conference. Proceedings, 1997.

[4] Malerba, C., Valentini, M., Ricardo, C.A., Rinaldi, A., Cappelletto, E., Scardi, P. \& Mittiga, A., Blistering in $\mathrm{Cu} 2 \mathrm{ZnSnS} 4$ thin films: correlation with residual stresses. Materials \& Design, 108, pp. 725-735, 2016. https://doi.org/10.1016/j.matdes.2016.07.019

[5] Huang, S. \& Zhang, X., Gradient residual stress induced elastic deformation of multilayer MEMS structures. Sensors and Actuators A: Physical, 2007, 134(1), pp. $177-185$.

https://doi.org/10.1016/j.sna.2006.05.026

[6] Zhang, X., Xu, B.S., Wang, H.D. \& Wu, Y.X., An analytical model for predicting thermal residual stresses in multilayer coating systems. Thin Solid Films, 488(1), pp. 274-282, 2005. https://doi.org/10.1016/j.tsf.2005.04.027

[7] Widjaja, S., Limarga, A.M. \& Yip, T.H., Modeling of residual stresses in a plasmasprayed zirconia/alumina functionally graded-thermal barrier coating. Thin Solid Films, 434(1), pp. 216-227, 2003. https://doi.org/10.1016/S0040-6090(03)00427-9

[8] Soliman, H. \& Waheed, A., Effect on differential thermal expansion coefficient on stresses generated in coating. Journal of Materials Science \& Technology (China) (USA), 15(5), pp. 457-462, 1999.

[9] Podstrigach, Y.S. \& Shevchuk, P., Effect of surface layers on diffusion processes and the resulting stress state in solids. Materials Science, 3(5), pp. 420-426, 1968. https://doi.org/10.1007/BF00716058

[10] Nguyen, T., Hubbard, J. \& Pommersheim, J., Unified model for the degradation of organic coatings on steel in a neutral electrolyte. JCT, Journal of Coatings Technology, 68(855), pp. 45-56, 1996.

[11] Deshpande, R., Cheng, Y.T. \& Verbrugge, M.W., Modeling diffusion-induced stress in nanowire electrode structures. Journal of Power Sources, 195(15), pp. 5081-5088. https://doi.org/10.1016/j.jpowsour.2010.02.021

[12] Nazir, M., Khan, Z.A., Saeed, A. \& Stokes, K., A model for cathodic blister growth in coating degradation using mesomechanics approach. Materials and Corrosion, 2015. https://doi.org/10.1016/j.engfailanal.2016.02.014

[13] Nazir, M., Khan, Z.A., Saeed, A. \& Stokes, K., A predictive model for life assessment of automotive exhaust mufflers subject to internal corrosion failure due to exhaust gas condensation. Engineering Failure Analysis, 63, pp. 43-60, 2016.

https://doi.org/10.1016/j.engfailanal.2016.02.014

[14] Nazir, M., Khan, Z.A., Saeed, A. \& Stokes, K., Modeling the effect of residual and diffusion-induced stresses on corrosion at the interface of coating and substrate. Corrosion, 72(4), pp. 500-517, 2015.

https://doi.org/10.1080/01694243.2015.1071023 
[15] Nazir, M., Khan, Z.A. \& Stokes, K., A holistic mathematical modelling and simulation for cathodic delamination mechanism-a novel and an efficient approach. Journal of Adhesion Science and Technology, 29(22), pp. 2475-2513, 2015. https://doi.org/10.1016/j.engfailanal.2016.07.003

[16] Nazir, M., Khan, Z.A. \& Stokes, K., Analysing the coupled effects of compressive and diffusion induced stresses on the nucleation and propagation of circular coating blisters in the presence of micro-cracks. Engineering Failure Analysis, 70, pp. 1-15, 2016. https://doi.org/10.1080/01694243.2015.1026870

[17] Nazir, M., Khan, Z.A. \& Stokes, K., Optimisation of interface roughness and coating thickness to maximise coating-substrate adhesion-a failure prediction and reliability assessment modelling. Journal of Adhesion Science and Technology, 29(14), pp. 1415$1445,2015$. https://doi.org/10.1080/01694243.2015.1026870

[18] Khan, Z.A., Chacko, V. \& Nazir, H., A review of friction models in interacting joints for durability design. Friction, 2017.

[19] Khan, Z.A., Pashaei, P., Bajwa, R., Nazir, H. \& Cakmak, M., Fabrication and characterisation of electrodeposited and magnertron-sputtered thin films. International Journal of Computational Methods \& Experimental Measurements, 3(2), pp. 165-174, 2015. https://doi.org/10.2495/CMEM-V3-N2-165-174

[20] Nazir, M. and Khan, Z., A review of theoretical analysis techniques for cracking and corrosive degradation of film-substrate systems. Engineering Failure Analysis, 2016.

[21] Nazir, M., Khan, Z.A. \& Stokes, K., A unified mathematical modelling and simulation for cathodic blistering mechanism incorporating diffusion and fracture mechanics concepts. Journal of Adhesion Science and Technology, 29(12), pp. 1200-1228, 2015. https://doi.org/10.1080/01694243.2015.1022496

[22] Nazir, M.H. \& Khan, Z., Maximising the interfacial toughness of thin coatings and substrate through optimisation of defined parameters. International Journal of Computational Methods and Experimental Measurements, 3(4), pp. 316-328, 2015. https://doi.org/10.2495/cmem-v3-n4-316-328

[23] Saeed, A., Khan, Z.A., Nazir, H., Hadfield, M. \& Smith, R., Research impact of conserving large military vehicles through a sustainable methodology. International Journal of Heritage Architecture, 1(2), pp. 267-274, 2017. https://doi.org/10.1016/j.matchemphys.2016.04.068

[24] Saeed, A., Khan, Z.A. \& Nazir, M.H., Time dependent surface corrosion analysis and modelling of automotive steel under a simplistic model of variations in environmental parameters. Materials Chemistry and Physics, 178, pp. 65-73, 2016. https://doi.org/10.3390/su71215825

[25] Saeed, A., Khan, Z.A. \& Nazir, M.H., An optimised approach of protecting and sustaining large vehicle system. Sustainability, 7(12), pp. 16451-16464, 2015. https://doi.org/10.3390/su71215825

[26] Lide, D.R., CRC Handbook of Chemistry and Physics, CRC Press, 85, 2004.

[27] Choi, S.R., Hutchinson, J.W. \& Evans, A., Delamination of multilayer thermal barrier coatings. Mechanics of Materials, 31(7), pp. 431-447, 1999. https://doi.org/10.1016/S0167-6636(99)00016-2

[28] Yu, H.-H. \& Hutchinson, J.W., Influence of substrate compliance on buckling delamination of thin films. International Journal of Fracture, 113(1), pp. 39-55, 2002. https://doi.org/10.1023/A:1013790232359 